\title{
Effect of Unsteady Cavity on Propeller-induced Hydrodynamic Pressure
}

\author{
Noritane Chiba, Member* \\ Tetsuji Hoshino, Member*
}

\begin{abstract}
Summary
The fluctuating pressures were measured on a flat plate above the cavitating propeller and the behavior of unsteady cavity was observed in detail at the same time, in order to make clear what is essential to estimating the pressure signals in full scale from model experiments.

As a result, the shape of pressure signals became very close to that in full scale when the pressure impulses induced by collapse of unsteady cavity were superposed on the fluctuating pressure. Accordingly, collapse of unsteady cavity should be taken into account in model experiments in order to simulate the fluctuating pressure in full scale. Further studies are necessary on simulation of collapse of unsteady cavity or simulation of flow field in full scale.
\end{abstract}

\section{Introduction}

The prediction of propeller-induced hydrodynamic pressure is one of the most important problems to be solved in order to reduce the ship vibration.

In early studies on this problem, experimental and theoretical efforts were devoted to non-cavitating propellers.

Recently, it was found in full scale measurements $^{1-4)}$, that the fluctuating pressures in full scale were much larger than those in 'model experiments on non-cavitating propellers. The model experiments carried out at the same time with these full scale measurents and those in wide ranges by Van Oossanen and van der Kooij ${ }^{5)}$ made it clear that the cavitating propellers in non-uniform flow could increase the fluctuating pressure as large as experienced in full scale.

Huse $^{6)}$ calculated the fluctuating pressure induced by unsteady cavity, the volume of which was assumed to vary on the propeller blades as a sinefunction. The result of his calculation showed that the volume variation of the unsteady cavity could increase the fluctuating pressure by the same order as experienced in the above measurements.

Ohtaka ${ }^{7)}$ measured several years ago the fluctuating pressure on the single screw ships built in Nagasaki Shipyard, MHI. He obtained, although unpublished, quite different shapes of pressure signals from those induced by non-cavitating propellers. High positive pressure impulses which appeared in accordance with blade frequency, were superposed on the fluctuating pressures as shown in Fig. 1.

* Nagasaki Experimental Tank, MHI.
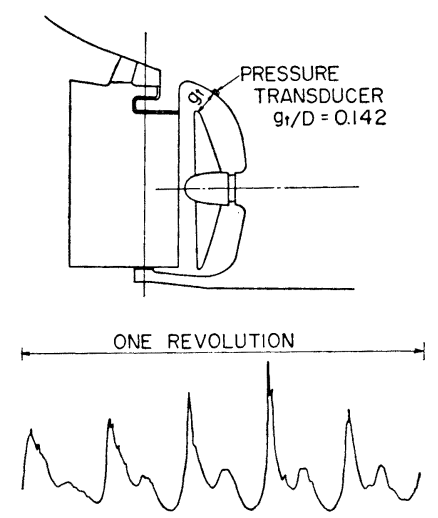

Fig. 1 Pressure fluctuation measured on the $50,000 \mathrm{DWT}$ tanker

Similar pressure signals were shown in the measurement on the Great Lakes Bulk Carrier4). These pressure impulses were supposed to be induced by unsteady cavity on the propeller. But the relation between the pressure signals and the behavior of unsteady cavity has not yet been examined and the pressure signals in full scale have not been correctly simulated in model experiments.

In the cavitation tunnel of Nagasaki Experimental Tank, MHI., propeller-induced hydrodynamic pressures were measured on the flat plate above the propeller and the behavior of unsteady cavity was observed in detail at the same time, in order to find out what is essential in model experiments to simulate correctly the pressure signals of full scale.

\section{Test Apparatus}

The measurements were carried out in the cavita- 

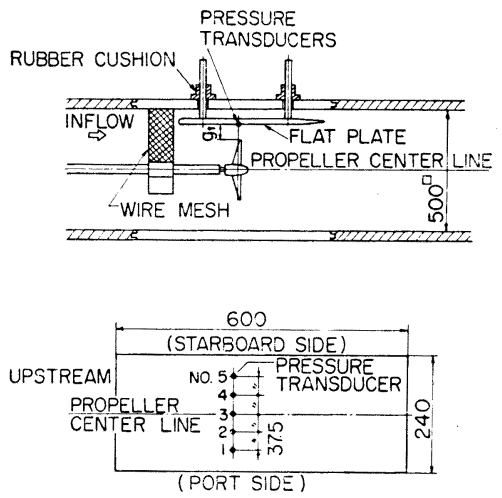

Fig. 2 Arrangement of test apparatus in cavitation tunnel

tion tunnel of Nagasaki Experimental Tank, MHI. The flat plate was set above the model propeller parallel to the propeller shaft as shown in Fig. 2. Five pressure transducers were mounted flush with the surface of the flat plate, with equal distance in transverse direction in the propeller disc plane. In order to avoid the effect of the vibration of the cavitation tunnel, rubber cushions were inserted between the supports of the flat plate and the tunnel wall.

The pressure transducers were $6 \mathrm{~mm}$ in diameter and of strain gauge type. The capacity was $2 \mathrm{~kg} / \mathrm{cm}^{2}$ and frequency characteristics was flat up to $20 \mathrm{kHz}$. The maximum response frequency of the amplifier used in the measurements was $10 \mathrm{kHz}$.

To make the non-uniform flow, a wire mesh was placed in the upstream of the propeller.

\section{Preliminary Test}

When fluctuating pressures induced by a propeller are measured in a cavitation tunnel, the measured signals are liable to be affected by the reflection of the pressure from the tunnel wall ${ }^{6}$.

In order to examine this influence, the fluctuating pressures induced by the two geometrically similar model propellers were measured in uniform flow. The smaller propeller was designated as Propeller A and the larger, as Propeller B, which had the standard diameter in our tunnel. The diameter of Propeller A is about 0.75 times as large as that of Propeller B. The principal details of both propellers are shown in Table 1 . The measurements

\begin{tabular}{|l|l|l|}
\hline CONDITION & $\begin{array}{l}\text { NON- } \\
\text { CAVITATING }\end{array}$ & $\begin{array}{c}\text { CAVITATING } \\
\left(\sigma_{n}=1.5\right)\end{array}$ \\
\hline PROPELLER A & ---- & $-\cdots-$ \\
\hline PROPELLER B & - & - \\
\hline
\end{tabular}

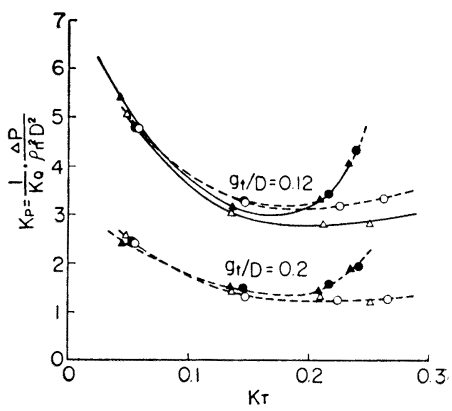

Fig. 3 Pressure amplitudes induced by the geometrically similar models on the top position of the propeller (No. 3 pressure transducer)

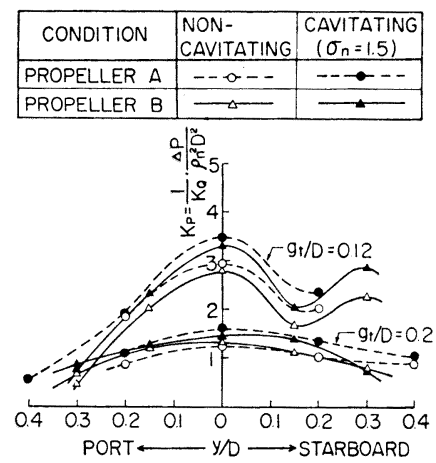

Fig. 4 Pressure amplitudes induced by the geometrically similar models in the transverse direction $\left(K_{T}=0.21\right)$

were made at two tip clearance ratios, i.e. $g_{t} / D=$ 0.12 and 0.20 , where $g_{t}$ is the distance from the propeller tip to the flat plate and $D$ is the diameter of the model propeller.

The measured amplitudes were made into the following non-dimensional form and compared in Fig. 3 and Fig. 4.

$$
K_{p}=\Delta P / K_{Q} \rho n^{2} D^{2}
$$

where

$\Delta P: \quad$ Pressure amplitude (peak to peak)

Table 1 Principal details of propellers

\begin{tabular}{l|c|c|c|c}
\hline & Propeller A & Propeller B & Propeller C & Propeller D \\
\hline Diameter (mm) & 186 & 250 & 250 & 250 \\
Pitch ratio & 0.727 & 0.727 & 0.637 & 0.689 \\
Expanded area ratio & 0.603 & 0.603 & 0.649 & 0.649 \\
Number of blades & 5 & 5 & 5 & 5 \\
\hline
\end{tabular}




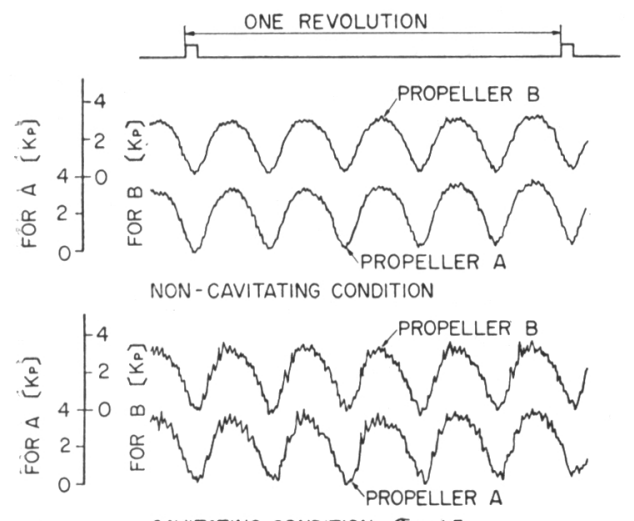

CAVITATING CONDITION, $\sigma_{n}=1.5$

Fig. 5 Pressure signals in uniform flow $\left(K_{T}=0.21, g_{i} / D=0.12\right)$

$K_{Q}$ : Torque coefficient of propeller $=$ Torque $/$ $\rho n^{2} D^{5}$

$\rho$ : Mass density of water

$n$ : Revolution of propeller

$D$ : Diameter of propeller

$K_{T}$ : Thrust coefficient of propeller $=$ Thrust/ $\rho n^{2} D^{4}$

The non-dimensional pressure amplitudes of both propellers agreed with each other within the accuracy of measurement both in non-cavitating and cavitating conditions. The shapes of pressure signals were also nearly the same, as shown in Fig. 5.

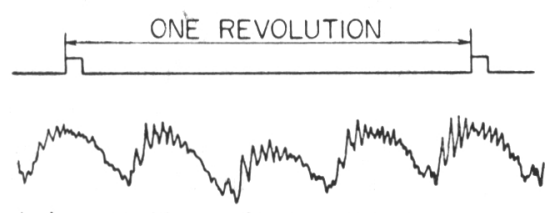

(A) $K_{T}=0.223$ NON-CAVITATING

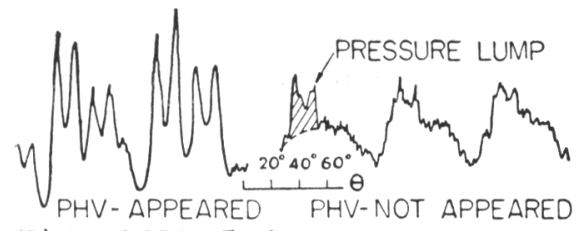

(B) $K_{T}=0.256, \sigma_{n}=2.0$

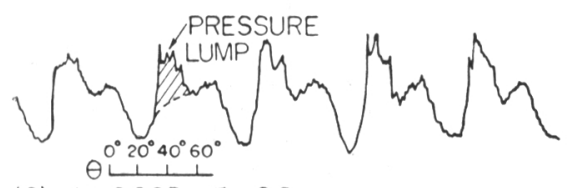

(C) $K_{T}=0.225, \sigma_{n}=2.0$
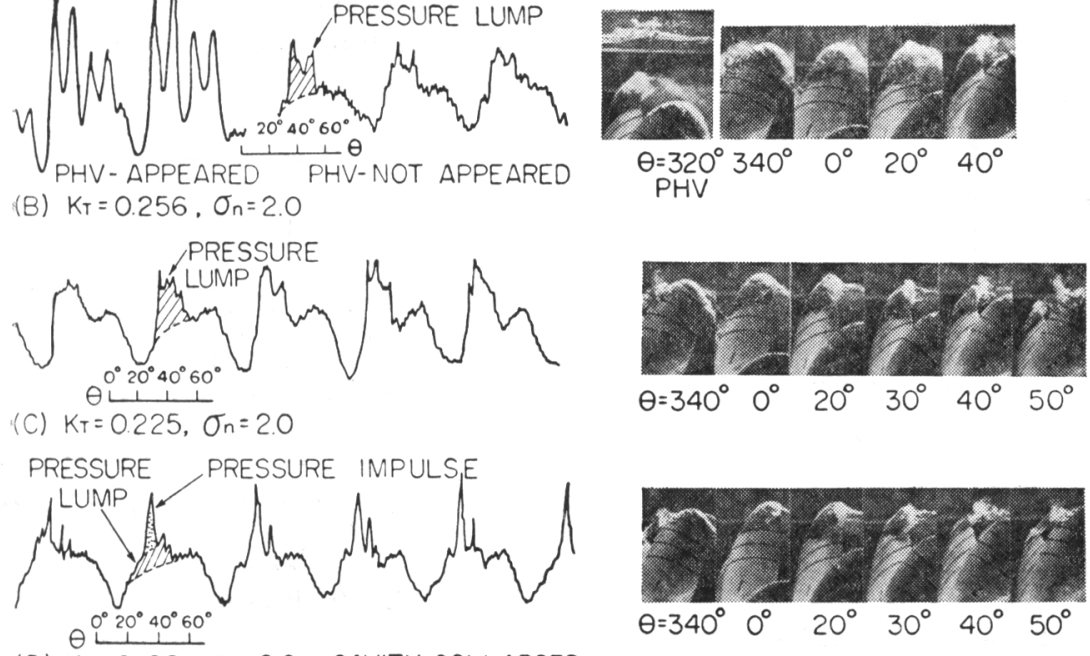

(D) $K_{T}=0.192, \sigma_{n}=2.0$ CAVITY COLLAPSED

Fig. 7 Pressure signals in non-uniform flow (No. 3 Pressure transducer)
This result showed that the tunnel wall did not affect substantially the measured pressure.

\section{Effect of Unsteady Cavity}

In order to make clear the effects of non-uniformity of flow and cavitation on the pressure signals induced by a propeller and to get the similar pressure signals as experienced in full scale measurements, the fluctuating pressures induced by Propeller $B$ were measured in the following conditions.

(a) uniform flow and non-cavitating condition

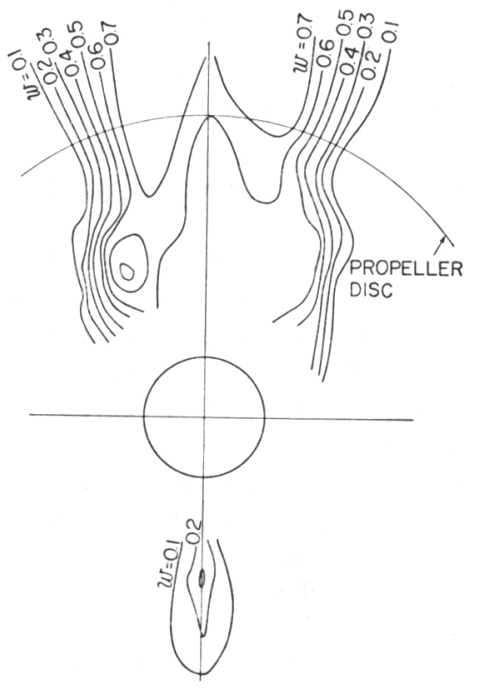

Fig. 6 Wake distribution 
(b) uniform flow and cavitating condition

(c) non-uniform flow and non-cavitating condition

(d) non-uniform flow and cavitating condition The tip clearance ratio $g_{t} / D$ was 0.12 . The wake distribution of the non-uniform flow adopted in this experiment is shown in Fig. 6. This wake distribution was expected to give a good correlation between ship and model in view of unsteady cavitation because the cavitation erosion patterns on the blades of model propellers operated in this wake distribution were well correlated to those in full scale ${ }^{8}$.

In uniform flow, the pressure signals were regular as shown in Fig. 5. The pressure peaks appeared in accordance with blade frequency. Cavitation in uniform flow did not affect the shape of pressure signals so much but increased the amplitudes as much as $30 \%$ in higher propeller load, as shown in Fig. 8.

In non-uniform flow and non-cavitating condition, the pressure signals were also regular as shown in Fig. 7(A). The amplitudes were increased as much as $60 \%$ in lower propeller load as shown in Fig. 8 . This may be due to the local increase of propeller load in high wake zone.

In non-uniform and cavitating condition, the pressure signals were quite different from those obtained in the preceding three conditions.

When the propeller load was very heavy, i.e. thrust coefficient $K_{T}=0.256$, the outer region of the propeller blade was covered with thick sheet cavity, changing its volume during one revolution. Propeller Hull Vortex (PHV) ${ }^{9)}$ appeared intermittently. PHV affected only a narrow area on the flat plate. The pressure amplitudes from one of the pressure transducers, where $\mathrm{PHV}$ reached, became several times higher than those in the absence of PHV, as

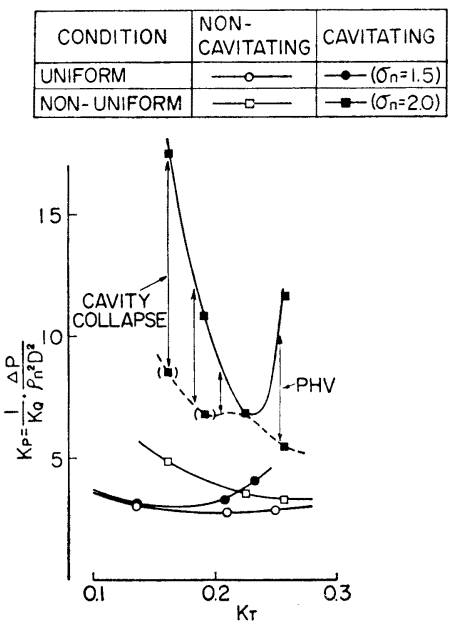

Fig. 8 Pressure amplitudes in nonuniform and cavitating conditions (Propeller B) shown in Fig. 7(B) and Fig. 8. In the absence of $\mathrm{PHV}$, positive pressure lumps were superposed on the regular pressure signals in accordance with blade frequency, as shown in Fig. 7(B). These pressure lumps appeared in the phase from $\theta=30^{\circ}$ to $50^{\circ}$, where $\theta$ is the angular position of propeller blade measured from the top position. In these phases, the cavity volume was decreasing as shown in Fig. 7(B). The pressure amplitude was higher than those in the preceding three conditions even in the absence of PHV as shown in Fig. 8.

When the propeller load was reduced to the value $K_{T}=0.225$, the sheet cavity became small except in the high wake region, where the cavity volume changed more violently than in the preceding propeller load. PHV disappeared. The pressure lumps became higher owing to the higher rate of decrease of cavity volume, as shown in Fig. 7(C) and 8. The phase of the pressure lumps was also in accordance with that of decrease of cavity volume.

When the propeller load was reduced further to the value $K_{T}=0.192$ which corresponded to the operating condition of Propeller $B$, the shape of pressure signals became very close to those in full scale. The unsteady cavity appeared only when the blade passed through the high wake region. PHV did not appear. As shown in Fig. $7(D)$, the volume of unsteady cavity changes much more violently and the collapse of unsteady cavity occurred. A positive high pressure impulse was superposed on each pressure lump. The pressure impulse made the pressure amplitude much higher as shown in Fig. 8. The pressure amplitudes, which were obtained ignoring the pressure impulses, were also plotted in Fig. 8, as designated by ( $\mathbf{(})$. These amplitudes in Fig. 8 corresponded to the pressure lumps induced by the volume change of unsteady cavity and can be related to the fluctuation pressure calculated by $\mathrm{Huse}^{6)}$. Fig. 9 shows. for example, that the pressure impulses occurred

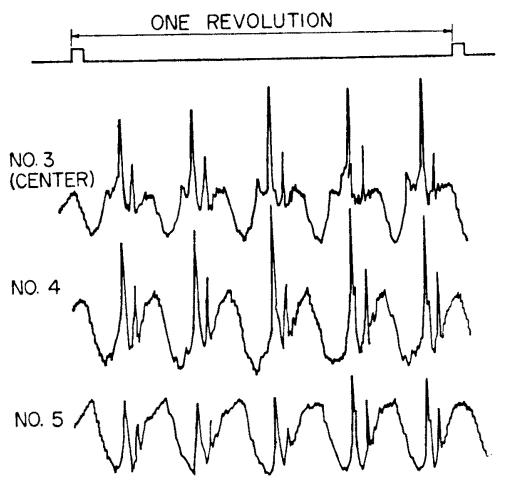

Fig. 9 Pressure impulses induced by collapse of unsteady cavity $\left(K_{T}=0.162, \sigma_{n}=2.0\right.$, Propeller B) 
in the same phase in transverse direction and the maximum amplitude occurred not on the center line transducer (No. 3), but on the transducer in the starboard side (No. 4). These facts indicated that the pressure impulses were induced by the collapse of unsteady cavity, which occurred in the starboard side.

The pressure impulses were examined further on Propeller C and D, because the behaviors of cavity collapse were different between them ${ }^{10)}$. The cavity collapse on Propeller $\mathrm{C}$ caused erosion on the blades because a part of cavity collapsed on the blades, but on the Propeller D, the collapse of cavity occurred as a whole in the tip vortex away from the blade. These be haviors of unsteady cavities were clearly shown in Fig. 10, together with the pressure signals measured at the same time. Fig. 10 shows that the pressure impulse occurred at the moment when the unsteady cavity became minimum in volume and like a cloud, that is collapse. This fact confirmed that the pressure impulse was induced by the collapse of unsteady cavity. Further, as the shape of pressure signals were nearly the same on both propellers, it may be true that the pressure impulse is related directly to cavity collapse and does not depend upon the place of its collapse.

Thus, the pressure signals similar to those in full scale were obtained in the cavitation tunnel. These pressure signals were found to be composed of three components. The first was the regular pressure signal induced by load and thickness of the propeller blades. The second was the pressure lump induced by the volume change of unsteady cavity. The third was the pressure impulse induced by the collapse of unsteady cavity. Accordingly, to simulate correctly the fluctuating pressure in full scale, it is essential to take into account the effect of unsteady cavity, especially the pressure impulse induced by collapse of unsteady cavity.

\section{Conclusion}

In order to find out what is essential to simulating correctly the pressure signals induced by full scale propellers in model experiments, the fluctuating pressures were measured on a flat plate above the cavitating propeller and the behavior of unsteady cavity was observed in detail at the same time.

As a result, the pressure signals in model experiments became very close to those in full scale measurements when the pressure impulses were induced by collapse of unsteady cavity. Therefore, it was made clear that the collapse of unsteady cavity should be taken into account in model experiments in order to estimate correctly the pressure signals in full scale.

Further studies are necessary on simulating the collapse of unsteady cavity or on simulating the flow field in full scale in model experiments.

\section{Acknowledgement}

The authors wish to express their gratitude to Mr. K. Ohtaka, Manager of Vibration Research Laboratory of Nagasaki Technical Institute, who kindly allowed to use his invaluable pressure records in full scale measurements, and to Mr. K. Tamura, Manager of Resistance and Propulsion Research Laboratory and Mr. H. Tanibayashi, Manager of Ship Experimental Tank, who encouraged them to complete this paper. Further the authors would like to thank the members of Nagasaki Experimental Tank for their cooperations.

\section{References}

1) Johnsson, C.A. and S $\phi$ ntvedt, T.: Propeller Excitation and Response of 230000 TDW Tankers, Swedish State Shipbuilding Experimental Tank, Publ. No. 70, (1972).

2) Takahashi, H.: On Propeller Vibratory Forces of the Container Ship-Correlation between Ship and Model, and the Effect of Flow Control Fin on Vibratory Forces, Ship Research Institute, Paper No. 44, (1973).

3) Weitendorf, E.A.: Vergleich von Propellererregten Druckschwankungen für Modell und Großausführung am Beispiel des Frachtschiffes 'Hornmeer', Schiff und Hafen, Jahrgang 25, Heft 5, (1973).

4) Kooij, J. van der and Jonk, A.: Propellerinduced Hydrodynamic Hull Forces on a Great Lakes Bulk Carrier. Results of Model Tests and Full Scale Measurements, Symposium on High Powered Propulsion of Large Ships, Wageningen, (1974).

5) Oossanen, P. van and Kooij, J. van der: Vibratory Hull Forces Induced by Cavitating Propellers, Journal of the Royal Institution of Naval Architects, Vol. 116, No. 2, (Apr. 1973).

6) Huse, E.: Effect of Cavitation on Propellerinduced Vibratory Forces, 14th ITTC Report of Propeller Committee, (1975).

7) Ohtaka, K.: Some Problems on the Stern Vibration of Ships (in Japanese), A Monthly Journal of the Society of Naval Architects of Japan, No. 554, (Aug. 1975).

8) Tanibayashi, H. and Nakanishi, M.: On the Method of Cavitation Tests for Prediction of Tip Erosion of Propeller, Journal of the Society of Naval Architects of Japan, Vol. 133, (1973).

9) Huse, E.: Propeller-Hull Vortex Cavitation, International Shipbuilding Progress, Vol. 19, No. 212, (Apr. 1972).

10) Chiba, N.: Behavior of Cavity Collapse as Cause of Cavitation Damage of Propeller Blades, ASME Symposium on Cavity Flow, Minneapolis, (1975). 

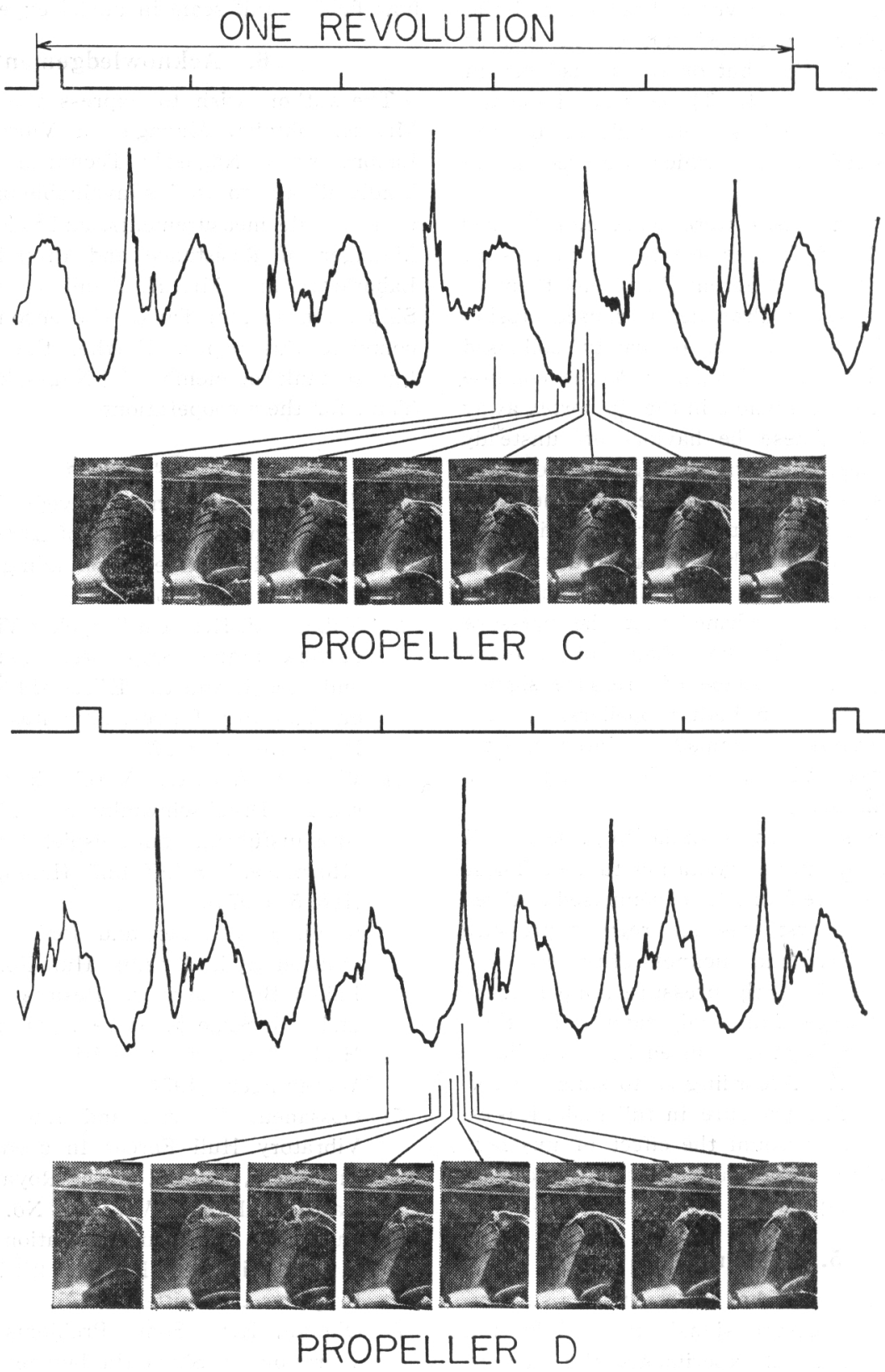

Fig. 10 Pressure impulse induced by collapse of unsteady cavity 